\title{
Phase retrieval and differential phase-contrast imaging with low-brilliance $\mathrm{X}$-ray sources
}

\author{
FRANZ PFEIFFER ${ }^{1 *}$, TIMM WEITKAMP², OLIVER BUNKK ${ }^{1}$ AND CHRISTIAN DAVID ${ }^{1}$ \\ ${ }^{1}$ Paul Scherrer Institut, 5232 Villigen PSI, Switzerland \\ ${ }^{2}$ Forschungszentrum Karlsruhe, 76021 Karlsruhe, Germany \\ *e-mail: franz.pfeiffer@psi.ch
}

$\mathbf{x}$ -ray radiographic absorption imaging is an invaluable tool in medical diagnostics and materials science. For biological tissue samples, polymers or fibre composites, however, the use of conventional X-ray radiography is limited due to their weak absorption. This is resolved at highly brilliant X-ray synchrotron or micro-focus sources by using phase-sensitive imaging methods to improve the contrast ${ }^{1,2}$. However, the requirements of the illuminating radiation mean that hard-X-ray phase-sensitive imaging has until now been impractical with more readily available $\mathrm{X}$-ray sources, such as $\mathrm{X}$-ray tubes. In this letter, we report how a setup consisting of three transmission gratings can efficiently yield quantitative differential phase-contrast images with conventional X-ray tubes. In contrast with existing techniques, the method requires no spatial or temporal coherence, is mechanically robust, and can be scaled up to large fields of view. Our method provides all the benefits of contrast-enhanced phase-sensitive imaging, but is also fully compatible with conventional absorption radiography. It is applicable to $\mathrm{X}$-ray medical imaging, industrial non-destructive testing, and to other low-brilliance radiation, such as neutrons or atoms.

In conventional X-ray imaging, contrast is obtained through the differences in the absorption cross-section of the constituents of the object. The technique yields excellent results where highly absorbing structures such as bones are embedded in a matrix of relatively weakly absorbing material, for example the surrounding tissue of the human body. However, in cases where different forms of tissue with similar absorption cross-sections are under investigation (for example, mammography or angiography), the X-ray absorption contrast is relatively poor. Consequently, differentiating pathologic from non-pathologic tissue in an absorption radiograph obtained with a current hospital-based X-ray system remains practically impossible for certain tissue compositions.

To overcome these limitations, several methods to generate radiographic contrast from the phase shift of X-rays passing through the sample have been investigated ${ }^{3-13}$. They can be classified into interferometric methods ${ }^{3,4}$, techniques using an analyser $^{5-7}$ and free-space propagation methods ${ }^{8-13}$. These methods differ vastly in the nature of the signal recorded, the experimental setup, and the requirements on the illuminating radiation. Because of the use of crystal optics, interferometric and analyser-based methods rely on a highly parallel and monochromatic X-ray beam. The required spatial and temporal coherence lengths ${ }^{14}$ are given by $\xi_{\mathrm{s}}=\lambda(\Delta \alpha / \alpha)^{-1}$ and $\xi_{\mathrm{t}}=\lambda(\Delta E / E)^{-1}$, where $\lambda$ is the wavelength, $\Delta \alpha / \alpha$ is the angular acceptance and $\Delta E / E$ is the energy band pass of the crystal optics. With typical values of $\Delta \alpha / \alpha \leq 10^{-4}$ and $\Delta E / E \leq 10^{-4}$, they range in the order of $\xi_{\mathrm{s}} \geq$ $10^{-6} \mathrm{~m}$ and $\xi_{\mathrm{t}} \geq 10^{-6} \mathrm{~m}$. Propagation-based methods can overcome the stringent requirements on the temporal coherence, and have been demonstrated to work well with a broad energy spectrum ${ }^{13,15}$ of $\Delta E / E \geq 10 \%\left(\xi_{\mathrm{t}} \sim 10^{-9} \mathrm{~m}\right)$. However, they still require a typical spatial coherence length of $\xi_{\mathrm{s}} \geq 10^{-6} \mathrm{~m}$, which is currently only available from micro-focus X-ray sources (with correspondingly low power) or synchrotrons. These constraints have, until now, hindered the final breakthrough of phase-sensitive X-ray imaging as a standard method for medical or industrial applications.

In this letter, we demonstrate how an alternative approach using a grating-based differential phase-contrast (DPC) setup can be efficiently used to retrieve quantitative phase images with polychromatic X-ray sources of low brilliance. Similarly to equivalent approaches in the visible light ${ }^{16,17}$ or soft X-ray range ${ }^{18}$, we recently showed that two gratings can be used for DPC imaging using polychromatic X-rays from brilliant synchrotron sources ${ }^{19,20}$.

Here, we describe how the use of a third grating allows for a successful adaptation of the method to X-ray sources of low brilliance. Our setup consists of a source grating $\mathrm{G}_{0}$, a phase grating $\mathrm{G}_{1}$, and an analyser absorption grating $\mathrm{G}_{2}$ (Fig. 1a) with periods $p_{0}$, $p_{1}$ and $p_{2}$. The source grating, typically an absorbing mask with transmitting slits, placed close to the X-ray tube anode, creates an array of individually coherent, but mutually incoherent sources. The ratio, $\gamma_{0}$, of the width of each line source to the period $p_{0}$ should be small enough to provide sufficient spatial coherence for the DPC image formation process. More precisely, for a distance 
$d$ between $\mathrm{G}_{1}$ and $\mathrm{G}_{2}$ corresponding to the first Talbot distance ${ }^{20}$ of $d=p_{1}^{2} / 8 \lambda$, a coherence length of $\xi_{\mathrm{s}}=\lambda l / \gamma_{0} p_{0} \geq p_{1}$ is required, where $l$ is the distance between $\mathrm{G}_{0}$ and $\mathrm{G}_{1}$. With a typical value of a few micrometres for $p_{1}$, the spatial coherence length required is of the order of $\xi_{\mathrm{s}} \geq 10^{-6} \mathrm{~m}$, similar to the requirements of existing methods (see above). It is important to note, however, that a setup with only two gratings $\left(G_{1}\right.$ and $\left.G_{2}\right)$ already requires, in principle, no spatial coherence in the direction parallel to the grating lines, in contrast with propagation-based methods ${ }^{8-13}$.

As the source mask $\mathrm{G}_{0}$ can contain a large number of individual apertures, each creating a sufficiently coherent virtual line source, standard X-ray generators with source sizes of more than a square millimetre can be used efficiently. To ensure that each line source produced by $\mathrm{G}_{0}$ contributes constructively to the image-formation process, the geometry of the setup should satisfy the condition (Fig. 1b)

$$
p_{0}=p_{2} \times \frac{l}{d}
$$

It is important to note that the total source size $w$ only determines the final imaging resolution, which is given by $w d / l$. The arrayed source thus decouples spatial resolution from spatial coherence, and allows the use of X-ray illumination with coherence lengths as small as $\xi_{\mathrm{s}}=\lambda l / w \sim 10^{-8} \mathrm{~m}$ in both directions, if the corresponding spatial resolution $w d / l$ can be tolerated in the experiment. Finally, as a temporal coherence of $\xi_{\mathrm{t}} \geq 10^{-9} \mathrm{~m}(\Delta E / E \geq 10 \%)$ is sufficient ${ }^{20}$, we deduce that the method presented here requires the smallest minimum coherence volume $\xi_{\mathrm{s}} \times \xi_{\mathrm{s}} \times \xi_{\mathrm{t}}$ for phase-sensitive imaging if compared with existing techniques.

The DPC image-formation process achieved by the two gratings $G_{1}$ and $G_{2}$ is similar to Schlieren imaging ${ }^{21}$ and diffraction-enhanced imaging ${ }^{5-7}$. It essentially relies on the fact that a phase object placed in the X-ray beam path causes slight refraction of the beam transmitted through the object. The fundamental idea of DPC imaging depends on locally detecting these angular deviations (Fig. 1b). The angle, $\alpha$ is directly proportional to the local gradient of the object's phase shift, and can be quantified by ${ }^{21}$

$$
\alpha=\frac{\lambda}{2 \pi} \frac{\partial \Phi(x, y)}{\partial x},
$$

where $x$ and $y$ are the cartesian coordinates perpendicular to the optical axis, $\Phi(x, y)$ represents the phase shift of the wavefront, and $\lambda$ is the wavelength of the radiation. For hard X-rays, with $\lambda<0.1 \mathrm{~nm}$, the angle is relatively small, typically of the order of a few microradians.

In our case, determination of the angle is achieved by the arrangement formed by $\mathrm{G}_{1}$ and $\mathrm{G}_{2}$. Most simply, it can be thought of as a multi-collimator translating the angular deviations into changes of the locally transmitted intensity, which can be detected with a standard imaging detector. For weakly absorbing objects, the detected intensity is a direct measure of the object's local phase gradient $\mathrm{d} \Phi(x, y) / \mathrm{d} x$. The total phase shift of the object can thus be retrieved by a simple one-dimensional integration along $x$. As described in more detail in refs 19,20, a higher precision of the measurement can be achieved by splitting a single exposure into a set of images taken for different positions of the grating $G_{2}$. This approach also allows the separation of the DPC signal from other contributions, such as a non-negligible absorption of the object, or an already inhomogeneous wavefront phase profile before the object. Note that our method is fully compatible with conventional absorption radiography, because it simultaneously yields separate absorption and phase-contrast images, so that information is available from both.
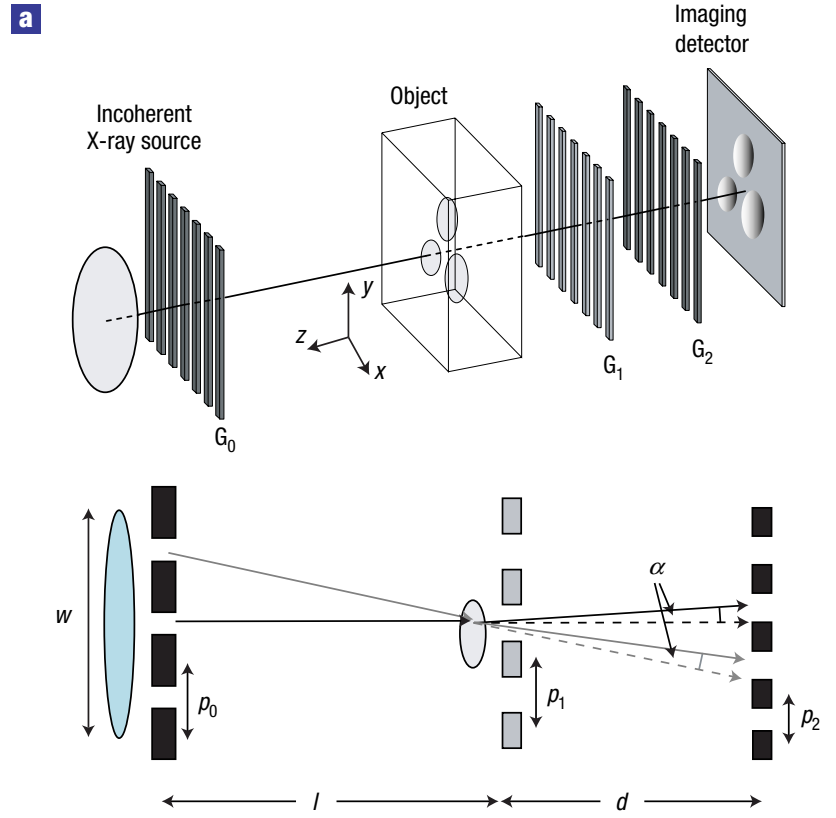

b

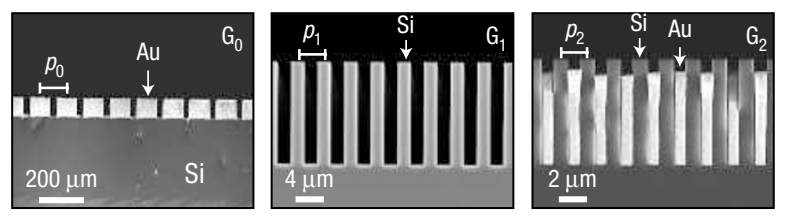

Figure 1 Talbot-Lau-type hard-X-ray imaging interferometer. a, Principle: the source grating $\left(\mathrm{G}_{0}\right)$ creates an array of individually coherent, but mutually incoherent sources. A phase object in the beam path causes a slight refraction for each coherent subset of $X$-rays, which is proportional to the local differential phase gradient of the object. This small angular deviation results in changes of the locally transmitted intensity through the combination of gratings $G_{1}$ and $G_{2}$. A standard $X$-ray imaging detector is used to record the final images. $\mathbf{b}$, Scanning electron micrographs of cross-sections through the gratings. The gratings are made from $\mathrm{Si}$ wafers using standard photolithography techniques, and subsequent electroplating to fill the grooves with gold $\left(\mathrm{G}_{0}\right.$ and $\left.\mathrm{G}_{2}\right)$.

Figure 2 shows processed absorption, DPC and reconstructed phase images of a reference sample containing spheres made of polytetrafluoroethylene (PTFE) and polymethylmethacrylate (PMMA). From the cross-section profiles (Fig. 2d), we deduce that $19 \pm 0.6 \%(7.0 \pm 0.6 \%)$ of the incoming radiation is absorbed in the centre of the PTFE (PMMA) sphere. By comparison with tabulated literature values ${ }^{22}$, a mean energy of the effective X-ray spectrum of $E_{\text {mean }} \approx 22.4 \pm 1.2 \mathrm{keV}(\lambda \approx 0.0553 \mathrm{~nm})$ is deduced. To discuss the results for the object's phase shift (Fig. 2c), we have to consider the real part of the refractive index, which, for X-rays, is typically expressed as $n=1-\delta$. For a homogenous sphere with radius $r$, the total phase shift through the centre of the sphere is $\Phi=4 \pi r \delta / \lambda$. The experimentally observed maxima of the integrated phase shifts are $\Phi_{\mathrm{PTFE}}=54 \pm 2 \pi$ and $\Phi_{\mathrm{PMMA}}=32 \pm 2 \pi$ (Fig. $2 \mathrm{f}$ ), from which we obtain: $\delta_{\mathrm{PTFE}}=9.5 \pm 0.8 \times 10^{-7}$ and $\delta_{\mathrm{PMMA}}=5.9 \pm 0.6 \times 10^{-7}$, which is in agreement with the values in the literature ${ }^{22}$. Note that because our method provides a quantitative measure of the absorption and integrated phase shift of the object in each pixel, the results can be used for further quantitative analysis, such as the reconstruction of a three-dimensional map of the real and 


\section{LETTERS}

a

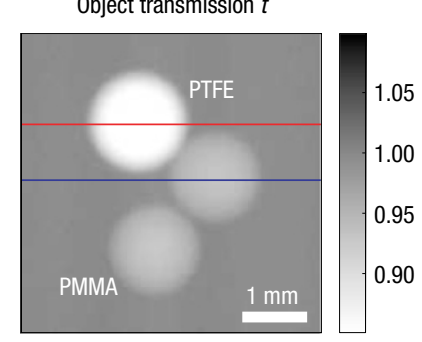

d

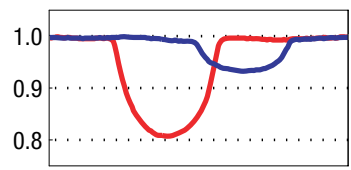

b

Phase gradient $\mathrm{d} \Phi / \mathrm{d} x\left(\pi \mathrm{mm}^{-1}\right)$

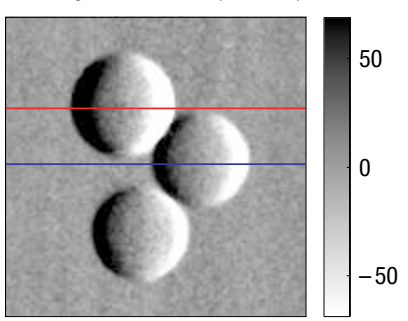

e

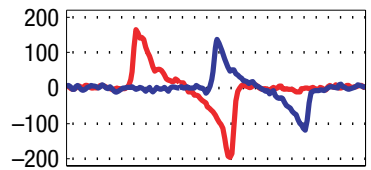

$\mathbf{G}$

Object phase shift $\Phi(\pi)$

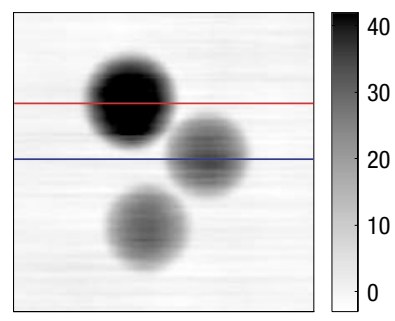

f 60

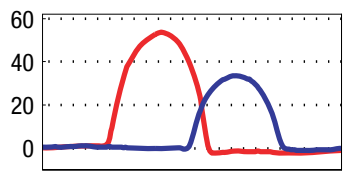

Figure 2 X-ray images and section profiles of a test sample containing one PTFE and two PMMA spheres, retrieved from image data recorded with a standard X-ray tube operated at $40 \mathrm{kV} / 25 \mathrm{~mA}$. a, Conventional X-ray transmission image. b, DPC image $\mathrm{d} \Phi / \mathrm{d} x$. c, Object phase shift $\Phi$ retrieved from $\mathrm{d} \Phi / \mathrm{d} x$ by integration. The images are represented on a linear greyscale. $\mathbf{d}-\mathbf{f}$, Section profiles through the corresponding image data of the PTFE (red) and the PMMA sphere (blue).
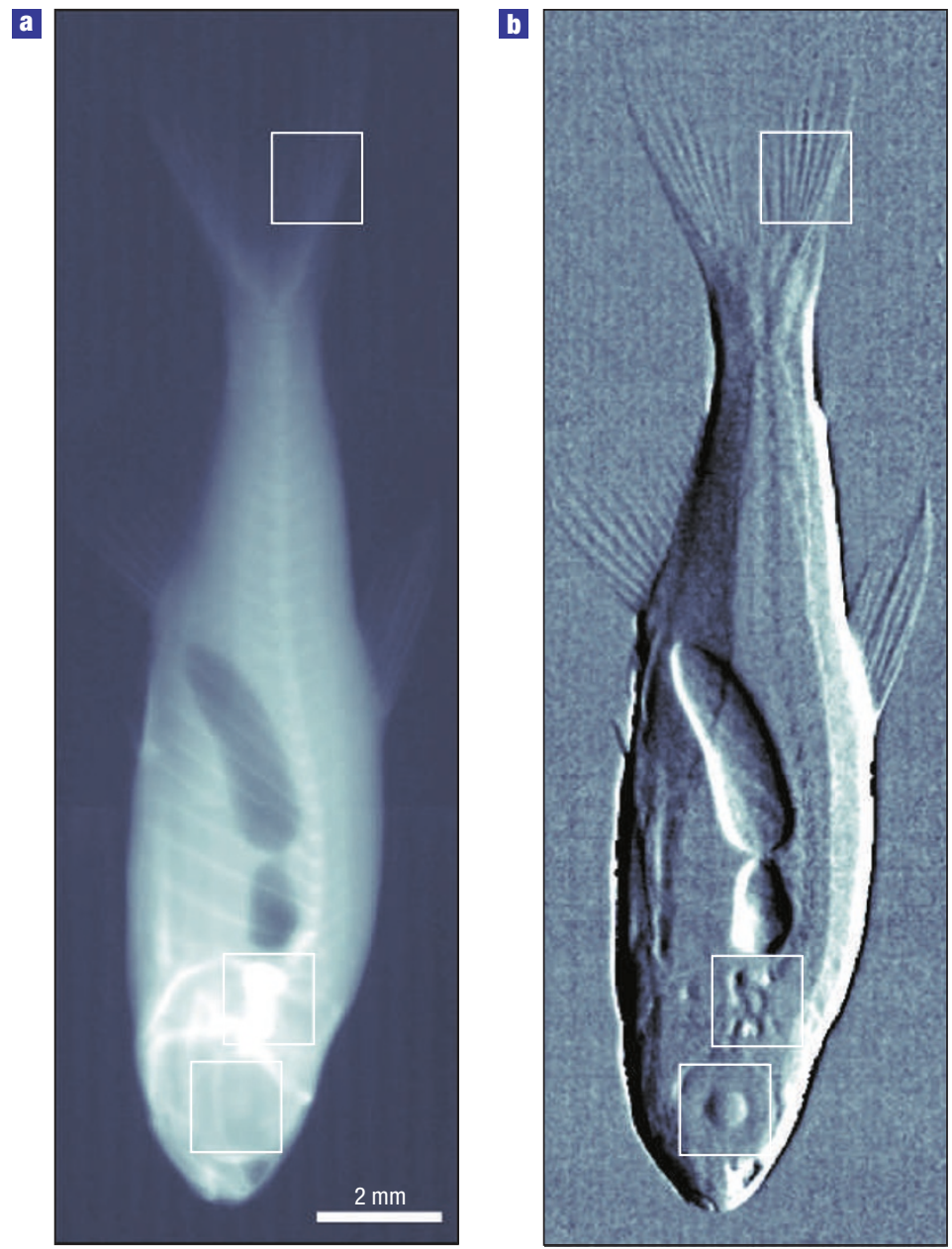
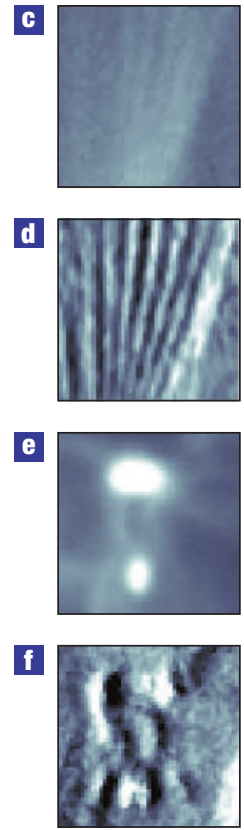

g

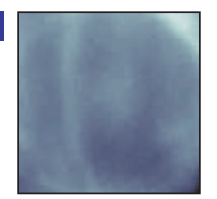

h

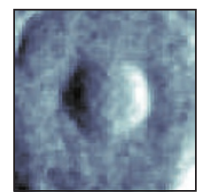

Figure $3 \mathrm{X}$-ray images of a small fish retrieved from image data recorded with a standard X-ray tube operated at $40 \mathrm{kV} / 25 \mathrm{~mA}$. a, Conventional X-ray transmission image. b, Differential phase-contrast image. $\mathbf{c}-\mathbf{h}$, Two-times magnified and contrast-optimized parts of the transmission (c,e,g) and the differential phase-contrast image $(\mathbf{d}, \mathbf{f}, \mathbf{h})$ : $\mathbf{c , d}$, parts of the tail fin; $\mathbf{e , f}$, the region around the otoliths; $\mathbf{g}, \mathbf{h}$, the eye of the fish. All images are represented on a linear greyscale. 
imaginary part of the object's refractive index using computerized tomography algorithms.

Figure 3 shows the results of applying our method to a biological object, a small fish (Paracheirodon axelrodi). The conventional X-ray transmission image is shown in Fig. 3a, whereas Fig. 3b contains a greyscale image of the corresponding DPC signal. As both images were extracted from the same data set, the total exposure time, and thus the dose delivered to the sample was identical for the two cases. The field of view is $20 \mathrm{~mm}$ (vertical) $\times 7 \mathrm{~mm}$ (horizontal). As expected, the skeleton of the fish and other highly absorbing structures, such as the calcified ear stones (otoliths) are clearly visible in the conventional radiograph (Fig. 3a,e). However, small differences in the density of the soft tissue, for example, the different constituents of the eye, are hardly visible in the conventional absorption image (Fig. 3g). In the corresponding DPC image (Fig. 3h), they are clearly visible. Likewise, the DPC image shown in Fig. $3 \mathrm{f}$ reveals complementary details of the soft tissue structure surrounding the otoliths, whereas only the highly absorbing structures are visible in the corresponding transmission image (Fig. 3e). Finally, we observe that, in particular, smaller structures with higher spatial frequencies, for example, the fine structure of the tail fin, are better represented in the DPC image (Fig. 3d) than in the corresponding absorption radiograph (Fig. 3c).

On the basis of these results, we conclude that the method presented here represents a major step forward in radiography, with standard X-ray tube sources that could provide all of the information imparted by conventional radiography, with additional information on soft tissue. This may prove to be of clinical importance, particularly in the detection of soft tissue pathologies. This hope is further supported by the fact that DPC methods generally exhibit advantages in the imaging of tumour masses, with relatively slow variations of the integrated phase shift compared with other phase-contrast imaging methods, for example, free-space propagation ${ }^{23}$. As the results were obtained with a standard, cheap and commercially available X-ray tube generator, we predict a widespread application of our method in areas where phase imaging is desirable, but is currently unavailable. For example, we believe that this method can readily be implemented without major changes to currently existing medical imaging systems, particularly in view of the ease of fabricating large-area gratings using standard photolithography, the high resistance of the method against mechanical instabilities, and the possibility of using detectors with large pixels and a large field of view (see Supplementary Information, Fig. S1). Furthermore, because the DPC image formation is not intrinsically coupled to the absorption of X-rays in the material, the radiation dose can potentially be reduced by using higher X-ray energies. Finally, these results open the way for phase-imaging experiments using other forms of radiation, for which only sources of relatively low brilliance currently exist, for example, beams of neutrons or atoms ${ }^{24}$

\section{METHODS}

The experiments were carried out on a Seifert ID 3000 sealed tube X-ray generator operated at $40 \mathrm{kV} / 25 \mathrm{~mA}$. We used a Mo line focus target FK 60-04, with a focus size of $8 \mathrm{~mm}$ (horizontal) $\times 0.4 \mathrm{~mm}$ (vertical). Because of the inclination of the target with respect to the optical axis of our setup of $\approx 6^{\circ}$, the effective source size in our setup was $0.8 \mathrm{~mm}$ (horizontal) $\times 0.4 \mathrm{~mm}$ (vertical).

The gratings were fabricated by a process involving photolithography, deep etching into silicon ${ }^{25}$ and, in the case of $\mathrm{G}_{0}$ and $\mathrm{G}_{2}$, electroplating with gold. They had periods of: $p_{0}=127 \mu \mathrm{m}, p_{1}=3.938 \mu \mathrm{m}$, and $p_{2}=2 \mu \mathrm{m}$. We currently use typical opening fractions of $\gamma_{0}=0.2-0.4\left(\mathrm{G}_{0}\right)$, and structure heights of the absorbing lines of $\sim 100 \mu \mathrm{m}\left(\mathrm{G}_{0}\right)$ and $10-15 \mu \mathrm{m}\left(\mathrm{G}_{2}\right)$. To date, we can fabricate suitable gratings with surface areas of up to $64 \mathrm{~mm} \times 64 \mathrm{~mm}$. The gratings were placed with their lines perpendicular to the optical axis of the setup and parallel to the vertical direction. The distances between the gratings were $l=1.765 \mathrm{~m}$ and $d=27.8 \mathrm{~mm}$. The detector was placed directly behind the last grating, $\mathrm{G}_{2}$.

The images were recorded using a $100-\mu \mathrm{m}$-thick yttrium aluminium garnet (YAG) fluorescence screen, with an optical lens system and a cooled charge-coupled device (FLI IMG 1001) equipped with a Kodak chip of $1,024 \times 1,024$ pixels (pixel size: $24 \mu \mathrm{m} \times 24 \mu \mathrm{m}$ ). The effective point-spread function was mainly determined by the thickness of the fluorescence screen to $\approx 30 \mu \mathrm{m}$. The exposure time for a single raw image was $40 \mathrm{~s}$. In total, $27 \mathrm{raw}$ images were recorded and processed to reduce the statistical and systematic errors of the measurement ${ }^{19,20}$. Note that the total exposure time can be greatly reduced by (1) using a more efficient detector, as our detection system only had an estimated efficiency of $\sim 1 \%$, (2) decreasing the distance between the source and the sample, and (3) using standard rotating anode $\mathrm{X}$-ray generators with a power of a few $\mathrm{kW}$.

The PTFE sphere used in the experiment had a density of $\rho=2.2 \mathrm{~g} \mathrm{~cm}^{-3}$ and a radius of $r=0.8 \mathrm{~mm}$. The corresponding values for the PMMA spheres are: $\rho=1.19 \mathrm{~g} \mathrm{~cm}^{-3}$ and $r=0.75 \mathrm{~mm}$.

\section{Received 15 December 2005; accepted 1 March 2006; published 26 March 2006.}

References

1. Fitzgerald, R. Phase-sensitive X-Ray imaging. Phys. Today 53, $23-27$ (2000).

2. Momose, A. Phase-sensitive imaging and phase tomography using X-ray interferometers. Opt. Express 11, 2303-2314 (2003).

3. Bonse, U. \& Hart, M. An x-ray interferometer with long separated interfering beam paths. Appl. Phys. Lett. 6, 155-156 (1965).

4. Momose, A., Takeda, T., Itai, Y. \& Hirano, K. Phase-contrast X-ray computed tomography for observing biological soft tissues. Nature Med. 2, 473-475 (1996).

5. Ingal, V. N. \& Beliaevskaya, E. A. X-ray plane-wave topography observation of the phase contrast from a non-crystalline object. J. Phys. D 28, 2314-2317 (1995).

6. Davis, T. J., Gao, D., Gureyev, T. E., Stevenson, A. W. \& Wilkins, S. W. Phase-contrast imaging of weakly absorbing materials using hard X-rays. Nature 373, 595-598 (1995).

7. Chapman, L. D. et al. Diffraction enhanced x-ray imaging. Phys. Med. Biol. 42, 2015-2025 (1997)

8. Snigirev, A., Snigireva, I., Kohn, V., Kuznetsov, S. \& Schelokov, I. On the possibilities of X-ray phase contrast microimaging by coherent high-energy synchrotron radiation. Rev. Sci. Instrum. 66, 5486-5492 (1995)

9. Wilkins, S. W., Gureyev, T. E., Gao, D., Pogany, A. \& Stevenson, A. W. Phase-contrast imaging using polychromatic hard X-rays. Nature 384, 335-337 (1996).

10. Cloetens, P. et al. Holotomography: Quantitative phase tomography with micrometer resolution using hard synchrotron radiation x rays. Appl. Phys. Lett. 75, 2912-2914 (1999).

11. Nugent, K. A., Gureyev, T. E., Cookson, D. F., Paganin, D. \& Barnea, Z. Quantitative phase imaging using hard X rays. Phys. Rev. Lett. 77, 2961-2964 (1996).

12. Mayo, S. C. et al. X-ray phase-contrast microscopy and microtomography. Opt. Express 11, 2289-2302 (2003)

13. Peele, A. G., De Carlo, F., McMahon, P. J., Dhal, B. B. \& Nugent, K. A. X-ray phase contrast tomography with a bending magnet source. Rev. Sci. Instrum. 76, 083707 (2005).

14. Als-Nielsen, J. \& McMorrow, D. Elements of Modern X-Ray Physics (Wiley, New York, 2001).

15. McMahon, P. J., Allman, B. E., Arif, M., Werner, S. A. \& Nugent, K. A. Quantitative phase radiography with polychromatic neutrons. Phys. Rev. Lett. 91, 145502 (2003).

16. Keren, E. \& Kafri, O. Diffraction effects in moire deflectometry. J. Opt. Soc. Am. A 2, 111-120 (1985).

17. Kafri, O. \& Glatt, I. The Physics of Moire Metrology (Wiley, New York, 1990).

18. Ress, D. et al. Measurement of laser-plasma electron density with a soft $\mathrm{x}$-ray laser moire deflectometer. Science 265, 514-517 (1994).

19. Weitkamp, T. et al. Hard X-ray phase imaging and tomography with a grating interferometer. Proc. SPIE 5535, 137-142 (2004)

20. Weitkamp, T. et al. Quantitative X-ray phase imaging with a grating interferometer. Opt. Express 13 6296-6304 (2005).

21. Born, M. \& Wolf, E. Principles of Optics (Pergamon, Oxford, 1980).

22. Henke, B. L., Gullikson, E. M. \& Davis, J. C. X-ray interactions: photoabsorption, scattering transmission, and reflection at $\mathrm{E}=50-30000 \mathrm{eV}, \mathrm{Z}=1-92$. At. Data Nucl. Data Tables 54, 181-342 (1993).

23. Pagot, E. et al. Quantitative comparison between two phase contrast techniques: diffraction enhanced imaging and phase propagation imaging. Phys. Med. Biol. 50, 709-724 (2005).

24. Keith, D. W., Ekstrom, C. R., Turchette, Q. A. \& Pritchard, D. E. An interferometer for atoms. Phys. Rev. Lett. 66, 2693-2696 (1991).

25. David, C., Ziegler, E. \& Nöhammer, B. Wet-etched diffractive lenses for hard X-rays. J. Synchrotron Radiat. 8, 1054-1055 (2001).

\section{Acknowledgements}

We gratefully acknowledge the assistance of C. Grünzweig in the measurements and P. R. Willmott for fruitful discussions.

Correspondence and requests for materials should be addressed to F.P.

Supplementary Information accompanies this paper on www.nature.com/naturephysics.

Competing financial interests

The authors declare that they have no competing financial interests.

Reprints and permission information is available online at http://npg.nature.com/reprintsandpermissions/ 